\title{
Boccaccio tra Dante e Cino
}

Nella sua monografia sul Decameron Mario Baratto illustra eloquentemente una caratteristica fondamentale dell'opera. In essa può accadere non solo che il discorso generi l'azione, ma che il discorso stesso costituisca l'unica azione di reale interesse narrativo: "[L]a retorica comica, la tecnica del discorso, l'articolazione di un dialogo possono non solo determinare la svolta di una vicenda, essere funzionali a un personaggio, ma porsi come l'effettivo nucleo strutturale di una novella" (289).

Sono nozioni verificabili nella novella di Zima (3.5), dove l'azione è interamente collegata alla presenza e assenza di discorso, sviluppandosi e compiendosi tutta nel monologo del protagonista e poi nel finto dialogo che egli inventa per ovviare al forzato silenzio della donna amata. La rubrica mette in chiaro fin dall'inizio che ci troviamo di fronte a una commedia di parola e silenzio: ${ }^{1}$

Il Zima dona a messer Francesco Vergellesi un suo pallafreno, e per quello con licenza di lui parla alla sua donna; e ella tacendo, egli in persona di lei si risponde, e secondo la sua risposta poi l'effetto segue. (3.5.1)

Francesco Vergellesi, ricco ma avaro gentiluomo di Pistoia, vuole un palafreno da sfoggiare durante il suo imminente soggiorno a Milano come podestà. Il miglior cavallo di Pistoia appartiene a Ricciardo detto il Zima, un giovane e ricco popolano innamorato della bella moglie di messer Francesco. Il soprannome di Ricciardo si riferisce al suo essere azzimato e la ricercatezza di Zima si rivelerà un elemento non tanto ornamentale quanto funzionale nella struttura del racconto. Soccombendo all' avarizia messer Francesco chiede al giovane di vendergli il cavallo, sperando che questi sia disposto a disfarsene senza voler nulla in cambio per amore della donna. Zima, che da tempo cerca senza successo di conquistare le grazie della virtuosa moglie, risponde che il cavallo non è in vendita ma che lo cederà alla seguente condizione: "che io, prima che voi il prendiate, possa con la grazia vostra e in vostra presenzia, parlare alquante parole alla donna vostra, tanto da ogni uom separato che io da altrui che lei udito non sia" (3.5.7). 
Convinto di poter superare in astuzia il damerino, messer Francesco acconsente e subito dopo comanda alla moglie di ascoltare tutto ciò che Zima le dirà ma senza mai a sua volta proferir parola. Sebbene mortificata dal trovarsi coinvolta in tale transazione d'affari, la moglie ritiene di non avere scelta e obbedisce. Confermati gli accordi tra marito e spasimante, ha luogo l'incontro. Il giovane ricorda alla donna il proprio tenacissimo amore e la prega di abbandonare il suo riserbo. La dichiarazione di Zima è condotta secondo schemi retorici codificati: egli impiega sapientemente le convenzioni stililistiche e concettualli della letteratura di lode seduttiva, secondo una tradizione che da Andrea Cappellano passa attraverso Provenzali e Siciliani negli Stilnovisti. L'effetto del discorso sulla donna è tale che per la prima volta nella vita comincia a provare cosa sia l'amore. Fedele alla parola data non pronuncia una sola sillaba di replica al fiorito discorso, tuttavia "non poté perciò alcun sospiretto nascondere quello che volentieri rispondendo al Zima avrebbe fatto manifesto" (3.5.17). ${ }^{2}$

Dopo aver aspettato invano una risposta Zima intuisce lo stratagemma del marito e ricorre a un'audace e originale contromossa:

E cominciò in forma della donna, udendolo ella, a rispondere a se medesimo [...]. (3.5.18)

Parlando in persona della donna il giovane dice, in sostanza, di sapere dell'amore di Zima, ma di non aver fino ad allora ceduto al suo corteggiamento per non infangare il proprio buon nome. Questo non vuol dire che non corrisponda il sentimento: adesso che il marito deve recarsi fuori città è proprio il momento di mostrargli quanto lo ama. Promette infine che il loro amore sarà portato a "piacevole e intero comp'mento" (3.5.21) esponendo nei particolari come debbano incontrarsi una volta che il marito abbia lasciato Pistoia. Detto questo, Zima riprende a parlare in persona propria e, ringraziata la donna per le "sue" parole, promette di eseguire alla lettera le "sue" istruzioni. Quando il marito sarà partito, la moglie seguirà le istruzioni di Zima, cosicché i due riusciranno a incontrarsi non solo una ma piú volte e potranno continuare la loro tresca anche dopo il ritorno di messer Francesco da Milano.

Considerata la trama della novella ci si può ora chiedere: in che rapporto sta, rispetto alla logica narrativa, lo stratagemma della risposta a sé stesso? Si tratta di un nodo funzionale o di un mero elemento decorativo che, se da un lato "fa" la storia, rendendola memorabile, dall'altro la rende instabile? Perché il seduttore parla in persona della donna? Non potrebbe esprimersi direttamente, una volta constatato il silenzio imposto all'interlocutrice? Potrebbe la seduzione aver lieto fine anche senza il ricorso allo stratagemma? $\grave{E}$ possibile, ma non certo. Zima pensa forse che assumere l'identità di lei lo favorisca nell'opera di seduzione. Proprio grazie a un procedimento teatrale che consenta di sfoggiare originalità, ricercatezza di parola, eleganza di 
espressione - un'eleganza che sconfina nell'affettazione - la strategia può risultare vincente. ${ }^{3}$

Dire che egli pensa rende forse troppo semplicistico il processo che ha portato alla scelta di tale strategia. Forse essa è, se cosí possiamo dire, istintiva, in perfetto accordo con quanto già sappiamo della sua personalità.

Un amore infelice sembra avvicinarlo, inizialmente, a Federigo degli Alberighi o a Gentile Carisendi: ma la lindezza che è all'origine del soprannome [...] può anche ricordare un personaggio "molto assettatuzzo" (I 1,9) come Ser Cepperello, del quale sembra avere, in tutt'altra dimensione morale, pure il meticoloso temperamento di attore. (Baratto 291)

La sottile osservazione si rivela assai pertinente. $\grave{E}$ opportuno infatti rilevare che Boccaccio rende azzimato il protagonista per uno scopo narrativo preciso. Attraverso l'abbigliamento elegante e la cura quasi esagerata della persona l'autore vuole suggerire un carattere narcisistico e una preziosità di stile tali da giustificare il ricorso all'espediente teatrale della risposta a sé stesso, l'espediente cardine dell'intera novella. Un semplice Ricciardo sarebbe stato del tutto inadeguato. Un Ricciardo chiamato il Zima, un Ricciardo "dandy", invece, mette subito a tacere qualsivoglia dubbio circa la necessità logiconarrativa dell'espediente istrionico cui il protagonista ricorre. ${ }^{4}$

In un altro caso almeno, nell'opera del Boccaccio, un personaggio articola il dialogo con l'amante esprimendo anche le repliche di quest'ultimo. Si tratta del dialogo in absentia di Fiammetta con Panfilo, nella Elegia di madonna Fiammetta:

Poi lui immaginava tornato e meco fingendolo, molte cose gli dicea, e di molte il dimandava, e io stessa in suo luogo mi rispondea. (3.12.4)

Mentre la risposta a sé stessa di Fiammetta è parte della sua fantasticheria, poiché essa parla nel libro in prima persona ammettendo il lettore nel proprio monologo interiore, nel caso di Zima si ha una fantasticheria espressa ad alta voce. Zima mette in scena sogni a occhi aperti per convincere l'amata e darle istruzioni necessarie. Mentre inscena il proprio sogno, egli vuol credere, e ne ha ben ragione, di esprimere anche i desideri più riposti della donna. Egli sogna ad alta voce al posto suo, intende mostrarle come quello sia in realtà anche il suo (di lei) sogno.

Lo sfondo culturale di questa novella non è stato adeguatamente esplorato. I critici menzionano l'influenza di Andrea Cappellano (Branca in Boccaccio, Decameron 370, n. 6), ma anche se Boccaccio aveva presente il testo di Andrea, non pare che la novella si presenti come reverente tributo al grande manuale dell'amor cortese. Semmai essa sembra porsi in termini canzonatori rispetto al modello, grazie all'impiego di procedimenti di esagerazione, distor- 
sione e abbassamento. Il difetto incarnato da Francesco Vergellesi, l'avarizia, è oggetto di chiara e ripetuta condanna nel Cappellano: "Avaritiam sicut nocivam pestem effugias et eius contrarium amplectaris" (116). Tuttavia, la transazione d'affari inventata da messer Francesco appartiene a un universo immaginativo ben lontano dalla stilizzata antropologia di Andrea. E se da un lato Zima sembra possedere tutte le qualità dell'amante ideale del trattato, compresa una "copiosa sermonis facundia" (42), la sua forbita eloquenza diventa un'affettata bizarria. Si pensi a come risulta stravolto il consiglio di Andrea per l'amante che si trovi ad affrontare il silenzio ostinato della donna: "Sed si nimis ipsius mulieris loquendi differantur initia, post spatium moderatum sapienter in sermone prorumpas" (46). Zima segue sí questo consiglio ma con un eccentrico, personalissimo tocco, la risposta "in forma della donna", appunto. Queste rispondenze quasi parodiche, tuttavia, non basterebbero da sole a provare una derivazione della novella di Boccaccio dal trattato di Andrea.

Una prova testuale piú forte si trova nel dettato del ricatto morale di Zima:

[...] la quale [vita], se a' miei prieghi l'altiero vostro animo non s'inchina, senza alcun fallo verrà meno, e morrommi, e potrete esser detta di me micidiale. (3.5.13)

In un passo da una delle dichiarazioni del De Amore - un libro che è quasi interamente un campionario di discorsi per la seduzione - leggiamo:

[...] si me igitur tui amoris spe frustratum dimiseris, me protinus mortem subire compellis, cui tua postea nullatenus poterit prodesse medela, et ita poteris homicida vocari. (Andreas 94)

Una formula cruciale della dichiarazione amorosa, dunque, ("e potrete essere detta di me micidiale"), trova un antecedente pressoché identico nel latino di Andrea ("et ita poteris homicida vocari"). A questo punto si può ragionevolmente sostenere che il trattato di Andrea sia una delle fonti, diretta o mediata, della novella di Boccaccio, sia per il linguaggio che per la configurazione della storia. Ma si deve senz'altro guardare oltre.

Innanzitutto è possibile riconoscere nella retorica cortese del discorso seduttivo alcune voci poetiche della tradizione lirica in volgare. Può vedersi una movenza cavalcantiana, come è stato notato, in: "riconforterete gli spiriti miei, li quali spaventati tutti trieman al vostro cospetto" (3.5.15). ${ }^{5} \mathrm{E}$ si potrà soprattutto rilevare materiale di Cino da Pistoia più di quanto non sia stato fatto finora. Non dovrebbe sorprendere che il damerino pistoiese usi nella sua dichiarazione d'amore la poesia amorosa del suo concittadino. Se la poesia di Cino riecheggia davvero nel discorso di Zima, potrebbe trattarsi di un'allusione voluta da parte di Boccaccio, in quel che sarebbe un suo tipico gioco di riferimenti criptici. Non dimentichiamo che la musa di Cino, Selvaggia, è una Vergellesi. La scelta di un Vergellesi e di sua moglie come personaggi 
della novella potrebbe collegarsi alla autobiografia lirica di Cino.

Riporto qui di seguito raffronti testuali tra la dichiarazione d'amore di Zima e dichiarazioni d'amore rintracciabili nella poesia di Cino:

\section{1) Zima}

e sì come umilissimo servidor vi priego [...] che la vostra benignità sia tanta [...] che io [...] possa dire che, come per la vostra bellezza innamorato sono, cosí per quella aver la vita; (3.5.13)

Cino:

Vanne via, mia canzon, di gente in gente, tanto che la piú gentil donna trovi,

e priega che suoi novi

e begli occhi amorosi dolcemente

amici sian de' miei,

quando per aver vita guardan lei. (91.37-42)

2) Zima:

E lasciamo stare che la mia morte non vi fosse onore [...]. (3.5.14)

Cino:

Dovunque sono, sto suo [di amore]servitore e sempre pur mi fa di male in peggio;

ma se m'ancide nolli fie onore. (137.12-14)

3) Zima:

[...] la quale [vita], se a 'miei prieghi l'altiero vostro animıo non s'inchina, senza alcun fallo verrà meno $[\ldots] .(3.5 .13)$

Cino:

Or inchinate a si dolce preghiera; (125.45)

Appare evidente il calco sui versi operato dal Boccaccio nella scrittura della dichiarazione amorosa. Più oltre analizzeremo un sottotesto ciniano riguardante non lo stile ma la struttura narrativa della novella. Rimanendo ai raffronti testuali dati sopra, si osserverà che l'ultimo è doppiamente suggestivo. Quando dice "Or inchinate a sì dolce preghiera", Cino non si rivolge a Selvaggia, bensì a Dante, in una canzone, informata dalle nozioni-guida della Vita nuova, intesa a consolarlo della morte di Beatrice. Boccaccio trovava dunque in questa canzone lo spunto immediato per far interagire i testi di Cino con il prosimetro dantesco. Anche quest'ultima opera costituisce probabilmente un sottotesto della novella di Zima. La Vita nuova, infatti, può aver fornito al Boccaccio la sanzione letteraria per l'impiego della risposta a sé stesso.

La critica non ha finora trovato una fonte che spieghi il ricorso a questo espediente. Branca ricorda che il motivo del marito che vende la moglie a un 
rivale si trova in molte favole orientali, ma non c'è molto in queste storie che aiuti a comprendere come abbia funzionato l'inventio nella parte del Decameron che veniamo analizzando. Di maggiore interesse è invece il suggerimento, sempre del Branca, di considerare la tradizione del "contrasto d'amore" (Boccaccio, Decameron 368, n. 1). Possiamo senz'altro convenire che l'immaginazione del Boccaccio possa esser stata stimolata dalle forme dialogiche della poesia medievale, ma, di nuovo, la natura e l'origine della risposta a sé stesso non risultano adeguatamente chiosate fino a quando non si ricordi che le forme poetiche dialogiche potevano essere oggetto di una sia pur elementare messa in scena. ${ }^{6}$ È probabile che Boccaccio abbia percepito potenziale narrativo in una forma di poesia drammatica quale era il monologo dialogico:

Par «mime dialogué», ou par «monologue dialogué», nous désignons un genre dramatique qui ne suppose pas de mise en scène régulière, et qui se distingue du drame proprement dit, moins par la nuture des sujets, que par la façon de les traiter et de les représenter. Il est illustré par des pièces à plusiers personnages, que jouait un acteur unique, pourvoyant seul à tous les besoins de la représentation et remplissant à la fois tous les rôles. (Faral 238)

Il contrasto veniva rappresentato da un intrattenitore di professione, a volte aiutato da un collega, uomo o donna. Quest'aiuto, tuttavia, non era essenziale: "Dando vita alla forma del contrasto, è pressoché sicuro che il giullare si sdoppiasse, modificando il timbro della voce in corrispondenza dei diversi personaggi messi in scena, e potesse modificare gli atteggiamenti del volto e del corpo" (Suitner 140). ${ }^{7}$

Considerato il possibile stimolo di origine teatrale, veniamo alla convenzione retorica che ha probabilmente catalizzato il processo, forse solo in parte consapevole, attraverso il quale Boccaccio è pervenuto all'invenzione centrale della novella. Si tratta di una forma di discorso ipotetico di cui Dante offre almeno due esempi nella Vita nuova. Il primo è anche un esempio di pensiero drammatizzato, cioè la rappresentazione dell'attività mentale di un personaggio in forma dialogica. Nel capitolo XV il poeta-amante medita sugli effetti devastanti che la presenza di Beatrice ha operato sulle sue facoltà:

Appresso la nuova trasfigurazione mi giunse uno pensamento forte, lo quale poco si partia da me, anzi continuamente mi riprendea, ed era di cotale ragionamento meco: "Poscia che tu pervieni a cosí dischernevole vista quando tu se' presso questa donna, perché pur cerchi di vedere lei? Ecco che tu fossi domandato da lei: che avrestù da rispondere ponendo che tu avessi libera ciascuna tua vertude in quanto tu le rispondessi?» $\mathrm{E}$ a costui rispondea un altro, umile pensero, e dicea: «S'io non perdessi le mie virtudi, e fossi libero tanto che io le potessi rispondere, io le direi [...]». (15.1-2)

Tenendo presente sia la dinamica psicologica che il dettato di questo passo 
dantesco, torniamo ora alla novella. Dopo aver parlato al posto della donna, Zima riprende la propria identità e cosí risponde:

"Carissima donna, egli è per soverchia letizia della vostra buona risposta sí ogni mia vertú occupata, che appena posso a rendervi grazie formar la risposta; e se io pur potessi come io disidero favellare, niun termine è sí lungo che mi bastasse a pienamente potervi ringraziare come io vorrei e come a me far si conviene; [...]». (3.5.23)

Sia in Dante che in Boccaccio un'emozione fortissima causata dall'amata impaccia la favella dell'amante. Nell'un caso "ciascuna sua vertude" non è "libera", nell'altro "ogni mia vertù" è "occupata". Entrambe le occorrenze presentano un'ipotesi auspicabile di discorso non inibito ("se io [...] fossi libero tanto che io le potessi rispondere"; "se io potessi come pur disidero favellare"). Tuttavia per Dante il mancamento, descritto come un venir meno delle facoltà vitali - un fatto serissimo, dunque, non un espediente retorico dipende dal suo essere in presenza di Beatrice. Il personaggio boccacciano, invece, dà prova di sopportare eccellentemente gli effetti della presenza della donna, anzi, lungi dall'essere incapacitato e interdetto, mette in atto un'efficace strategia di seduzione, rivelando doti di attore brillante. Ecco perché non può che apparire ironica la sua dichiarazione di difficoltà a parlare. Perché sarebbe allora appena capace di parlare? Ma perché è felice. E perché è così felice? Perché la sua donna, o meglio la donna quale lui l'ha impersonata, gli si è finalmente mostrata pietosa ed egli non corre più il rischio di morire di amore non corrisposto.

Al contrario, nel capitolo XV della Vita nuova, come in quelli limitrofi, Dante non riesce a ottenere la pietà di Beatrice. A nulla serve che il poeta abbia messo a nudo la propria anima e che abbia confessato di essere vicino a morire per l'intensità del sentimento amoroso. Sono questi i frangenti in cui si estingue per sempre la volontà di Dante di usare la poesia come mezzo di persuasione ad amare. Nei capitoli XVII e XVIII il poeta prenderà piena coscienza dell'unica via di uscita dalla propria disperazione: poetare su un argomento nuovo e più nobile, la lode disinteressata di Beatrice. Dunque, nella novella del Boccaccio troviamo tracce di una intenzionale risposta alla Vita nuova di Dante: una risposta giocosa, in chiave leggera, forse un poco irriverente. ${ }^{8}$

Mario Baratto ha notato come il prosimetro dantesco sia rilevante per la novella che segue immediatamente quella di Zima, la storia napoletana della seduzione di Catella da parte di Ricciardo (3.6). In Dante il coro delle donne ha un ruolo decisivo nella scelta di intrattenere con Beatrice una relazione esclusivamente poetica, quindi d'intraprendere una poesia di pura lode (276). In Decameron 3.6, al contrario, le donne, compresa la "donna dello schermo", hanno ruolo decisivo nel far riuscire il piano di seduzione dell'amata architettato dall'amante. Boccaccio usa dunque il materiale cortese dantesco ad 
un livello più basso, adattandolo in un progetto di commedia erotica. Lo stesso può dirsi per la novella di Zima: qui, come poi nella novella successiva di Catella e Ricciardo, "[l]'etica cortese" di estrazione dantesca - ma non solo dantesca "[viene] trasferita nell'avventura erotica" (Baratto 278). È nella novella di Zima, tuttavia, che si avverte più fortemente e diffusamente la presenza di Dante.

Nel capitolo XXII della Vita nuova la situazione presenta, tra l'altro, un divieto a parlare. Dopo la morte del padre di Beatrice Dante incontra un gruppo di donne in lutto e, avendo udito la loro descrizione della pietosa vista di Beatrice in lacrime, è sopraffatto dal dolore. Il poeta stesso allora si offre come oggetto di commiserazione per le donne. Vorrebbe parlar loro, ma ne è impedito dalla regola sociale che prescrive l'assoluta segregazione tra i sessi in tali circostanze. I commenti delle donne sul dolore di Beatrice e sulla reazione del poeta stimolano il processo creativo:

Onde io poi, pensando, propuosi di dire parole acciò che degnamente avea cagione di dire, ne le quali parole io conchiudesse tutto ciò che inteso avea da queste donne; e però che volentieri l'averei domandate se non mi fosse stata riprensione, presi tanta matera di dire come se io l'avesse domandate ed elle m'avessero risposto. E feci due sonetti; che nel primo domando, in quello modo che voglia mi giunse di domandare; ne l'altro dico la loro risponsione, pigliando ciò ch'io udio da loro si come lo mi avessero detto rispondendo. (22.7-8)

Sia Dante che Zima devono affrontare un divieto di parola. Dante, impossibilitato a parlare con le donne dell'amata, inventa un ipotetico dialogo in versi, ponendo la domanda e fornendo poi la risposta; Zima dà al proprio monologo la forma di dialogo rispondendo al posto della donna: in entrambi i casi siamo nel regno del "come se" (De Robertis 165-74). Ma l'operazione di Dante è essenzialmente retrospettiva, poiché, proiettando nell'immaginazione, e poi in poesia, un evento che non ha potuto aver luogo nella realtà, i suoi sonetti non sono che una riscrittura idealizzata del passato. Zima, invece, teatralizzando la propria fantasticheria vuole conseguire un effetto immediato nella realtà: egli detta al presente e al futuro le regole del proprio desiderio. ${ }^{9}$

Sulla base delle prove raccolte finora sembra ragionevole stabilire una connessione tra l'espediente drammatico al centro della novella, la risposta a sé stesso, e gli episodi di discorso ipotetico che si trovano nella Vita nuova di Dante. Con tutto ciò, il testo di Dante rimane forse una fonte collaterale per il Boccaccio. Ritengo infatti che sia Cino ad esercitare l'impressione preponderante sulla struttura di Decameron 3.5. Un'analisi ravvicinata del suo sonetto Ora che rise lo spirito mio, non considerato finora, fornisce indicazioni preziose:

Ora che rise lo spirito mio, doneava il pensero entro lo core, 
e con mia donna parlando d'amore, sotto pietate si covria ' 1 disio:

perché là il chiama la follia ched io vo i[n]seguendo, e mostrone dolore, e par ch'i' sogni, e sia com'om ch'è fòre tutto del senno, e se stesso ha 'n oblio.

Per questo donear che fa 'l pensero, fra me medesmo vo parlando, e dico che 'l suo sembiante non mi dice vero quando si mostra di pietà nemico, ch'a forza par ched el si faccia fero: perch'io pur di speranza mi nutrico. (47)

Un primo evidentissimo collegamento per analogia verbale può istituirsi tra la chiusa del sonetto e una frase della prima parte della dichiarazione di Zima:

[...] e sì come umilissimo servidor vi priego, caro mio bene, e sola speranza dell'anima mia, che nell'amoroso fuoco sperando in voi si nutrica, che la vostra benignità sia tanta [...]. (3.5.13)

Boccaccio tuttavia non si è limitato a ricollocare un verso del sonetto nella dichiarazione di Zima: si può affermare che egli si sia appropriato dell'intero sonetto di Cino come nucleo narrativo su cui incentrare e da cui sviluppare l'intera novella. ${ }^{10}$ L'adattamento comporta la trasposizione del "doneare" ("doneava il pensero entro lo core") dallo spazio mentale a quello fisico. La conversazione mentale con la donna che ha luogo nel sonetto di Cino ("e con mia donna parlando d'amore") diventa una vera e propria conversazione in Boccaccio, poiché il protagonista parla d'amore alla propria donna in sua presenza. È questo un caso di concretizzazione degli stimoli immmaginativometaforici, un espediente simile all'abbassamento (in questo caso dall'ideale al reale) che è una costante nell'opera di Boccaccio.

Abbiamo visto come la conversazione che Zima si aspetta di avere con la donna diventi suo malgrado un monologo in cui l'amante è costretto a rispondere a sé stesso ("E cominciò in forma della donna, udendolo ella, $a$ rispondere a se medesimo"). Il suo parlare alla donna assomiglia dunque a un parlare a sé stesso e si può confrontare con quanto fa Cino nel sonetto: "fra me medesmo vo parlando". Quando Cino parla a sé stesso non fa altro che persuadersi che il fermo rifiuto opposto dalla donna sia solo di facciata: ${ }^{11}$

fra me medesmo vo parlando, e dico che "l suo sembiante non mi dice vero quando si mostra di pietà nemico, ch'a forza par ched el si faccia fero [...]. (10-13)

Anche Zima articola questa distinzione tra realtà e apparenza, tra interiorità 
e comportamento, per volgerla poi a proprio favore quando reinterpreta tendenziosamente passato e presente allo scopo di convincere la donna. Assumendo l'identità femminile nella seconda parte del suo strano monologare, accortamente afferma:

Tuttafiata, se dura e crudele paruta ti sono, non voglio che tu creda ch'io nell' animo stata sia quella che nel viso mi sono dimostrata; anzi, t'ho sempre amato e tenuto caro innanzi a ogni altro uomo, ma così mi è convenuto fare e per paura d'altrui e per servare la fama della mia onestà. (3.5.20)

Ci si domanda, infine, se e in che misura i versi 7-8 del sonetto di Cino possano aver stimolato l'immaginazione di Boccaccio. Non sarabbe procedimento insolito trasformare la convenzionale follia erotica della poesia cortese in episodio di comportamento stravagante. Davvero le parole "se stesso ha 'n oblio" ben descrivono la perdita della propria identità e l'assunzione di quella della donna da parte del damerino.

Si deve qui parlare un'ultima volta della predilezione boccacciana per l'abbassamento (di contenuto, stilistico, ecc.). Si può sostenere che con tale criterio non solo alcune situazioni della Vita nuova siano state cooptate nella novella di Zima, come si è visto, ma anche un importante tema trattato nel Convivio. Verso la fine della storia, quando il monologo/dialogo è finito e Zima prende congedo, Boccaccio inserisce una coda di dialogo tra marito e amante. Tronfio d'orgoglio per lo stratagemma che gli ha consentito di ottenere il cavallo senza subire egli stesso perdita alcuna, messer Francesco chiede al giovane se ritiene che lui, Francesco, sia stato ai patti. Zima risponde che non lo crede, poiché l'accordo prevedeva una conversazione con sua moglie, non con una statua di marmo. Deliziato, messer Francesco procede a ricordargli che il cavallo adesso è di sua proprietà:

A cui il Zima rispose: «Messer sì, ma se io avessi creduto trarre di questa grazia ricevuta da voi tal frutto chente tratto n'ho, senza domandarlavi ve l'avrei donato: e or volesse Idio che io fatto l'avessi, per ciò che voi avete comprato il cavallo e io non l'ho venduto». (3.5.28).

Questo ragionamento conclusivo in termini di compravendita richiama per contrasto la proposta iniziale di Zima, quando, nel principio della novella, il giovane si era rifiutato di considerare la transazione come commerciale: "Messer, se voi mi donaste ciò che voi avete al mondo, voi non potreste per via di vendita avere il mio pallafreno, ma in dono il potreste voi bene avere, quando vi piacesse, con questa condizione: che io prima che voi il prendiate possa con la grazia vostra e in vostra presenzia parlare alquante parole alla donna vostra" (3.5.7). Secondo le parole iniziali del protagonista, dunque, la transazione era vista come dono, non del tutto incondizionato, certo, e anzi 
stranamente condizionato, ma cionondimeno dono, non compravendita.

Come possiamo spiegare allora il mutamento intervenuto tra l'inizio della storia e la sua conclusione, tra la definizione dello scambio in termini di dono prima e in termini di compravendita poi? Una possibile risposta può venire dal Convivio. Nel capitolo I del suo prosimetro filosofico Dante parla delle caratteristiche dell'autentica liberalità. Una di esse è "sanza essere domandato lo dono, dare quello" (I.8.3). Ritengo che Boccaccio avesse in mente, con ogni probabilità, questo capitolo del Convivio al momento di concepire l'impianto della novella. Verso la fine del capitolo Dante afferma:

La terza cosa ne la quale si può notare la pronta liberalitade, si è dare non domandato: acciò che 'l domandato non è da una parte vertù ma mercatantia, però che lo ricevitore compera, tutto che 'l datore non venda. Per che dice Seneca che «nulla cosa più cara si compera che quella dove i prieghi si spendono». Onde acciò che nel dono sia pronta liberalitade e che essa si possa in esso notare, allora, s[e] conviene esser netto d'ogni atto di mercatantia, conviene esser lo dono non domandato. (1.8.16-17)

Cominciamo con l'evidente prelievo testuale. Sembra proprio che il modello della frase di Zima "voi avete comprato il pallafreno e io non l'ho venduto" sia stato il detto di Dante "però che lo ricevitore compera, tutto che 'l datore non venda". ${ }^{12}$ Tale riscontro non può che incoraggiare a una ricerca di aspetti più reconditi del processo di appropriazione.

Il contrasto pocanzi rilevato tra l'iniziale definizione dello scambio in termini di dono e la conclusiva in termini di compravendita, è più apparente che reale. Con la sua battuta finale ("voi avete comperato il pallafreno e io non l'ho venduto") Zima smaschera, sia pure surrettiziamente, la vera natura dell'iniziativa di messer Francesco. Il riferimento al passo in cui Dante parla dei doni richiesti, contenuto in quella battuta, rivela la vera natura dell'iniziale offerta di comprare il cavallo da parte di messer Francesco: non si trattava di una vera proposta commerciale quanto di un'indiretta richiesta di dono. Per capire fino in fondo l'uso che Boccaccio fa del passo dantesco, non dobbiamo dimenticare Seneca, l'auctoritas su cui Dante fonda il proprio ragionamento. L'argomentare di Dante procede così: quando si fa richiesta di un dono, non si è più nell'ambito della "vertù", bensi in quello della "mercatantia", perché anche se colui che fa il dono non effettua una vera e propria vendita, colui che riceve il dono invece compra, e compra a un prezzo altissimo: "Nulla cosa più cara si compera che quella dove i prieghi si spendono" (I.8.16). Ci si può chiedere allora se Zima, citando tra le righe Dante, non stia dicendo a messer Francesco: "certamente avete pagato un prezzo altissimo per la vostra richiesta: voi, stupido che siete, mi avete dato vostra moglie".

Può esserci ancora un'altra componente nel gioco di riferimenti criptici allestito dal Boccaccio. Per la chiosa dell'asserzione senechiana nel capitolo del Convivio citato sopra Dante rinvia all'ultimo capitolo del suo libro: "Perché sì 
caro costa quello che si priega, non intendo qui ragionare, perché sufficientemente si ragionerà ne l'ultimo trattato di questo libro" (1.8.18). Ma Dante non completò mai il Convivio, cosicché il lettore rimane privo della promessa spiegazione. Ritengo contemplabile l'ipotesi che Boccaccio non resistette alla tentazione di portare a termine ciò che a Dante non era riuscito di completare, considerando la propria novella di Zima come un'illustrazione della verità contenuta nella sentenza senechiana, e quindi come completamento di quel capitolo del Convivio. La novella di Zima mostra l'assunto generale secondo cui nulla s'acquista più caramente delle cose richieste, illustra cioè l'assunto tramite un caso particolare, il caso di messer Francesco che paga con la moglie la propria richiesta del palafreno. Certo, l'illustrazione boccacciana è giocosa, condotta a un livello di discorso più basso di quello del Convivio. Dante e Seneca intendono un costo morale, l'orgoglio ferito, l'umiliazione che comporta la richiesta di dono. In Boccaccio il costo appare in un contesto mercantile, in cui gli oggetti da perdere o da guadagnare sono concreti: una donna e un cavallo.

Sempre in connessione con la problematica del dono non si deve tralasciare quanto il pensiero cristiano ha espresso in tema di donne e silenzio, un argomento non sufficientemente indagato finora dalla critica. Se riandiamo al Vecchio e al Nuovo Testamento con questo tema in mente (cosí come a libri di devozione e a raccolte di exempla e di precetti), troveremo la nozione che una donna silenziosa è un dono del Signore (vedi, per esempio, Siracide 26.14). Anche una lettura giocosa di sentenze come questa potrebbe aver contribuito all'inventio della novella boccacciana.

In anni recenti si è ricorsi sempre più spesso alla nozione di parodia, assieme a quella di ironizzazione, per definire alcuni fenomeni di intertestualità e interdiscorsività ricorrenti nel Decameron, e molti studiosi hanno messo in luce la diffusa pratica di scrittura parodica del Boccaccio. ${ }^{13}$ È ormai ampiamente accettata la nozione che uno tra i più importanti bersagli, se non il più importante, dei giochi parodici che Boccaccio perpetra, a volte con intenzioni canzonatorie, a volte serissime, è la Divina Commedia. Non solo si può riscontrare nelle novelle la rielaborazione di singoli segmenti testuali danteschi, ma anche sostenere che l'intero Decameron sia strutturato secondo una modalità parodica sul poema di Dante. ${ }^{14}$ La Commedia, tuttavia, è lungi dal costituire l'unico oggetto delle strategie parodiche di Boccaccio. Nel suo saggio su ironia e parodia nel Decameron, Carlo Delcorno espone concisamente la grande varietà dei testi parodiati nella scrittura decameroniana:

La novella boccacciana [...] è in primo luogo la riscrittura, sempre tenden-zialmente parodistica, dei più diversi generi letterari: antichi e medievali, orali e scritti, in prosa e in versi. [Boccaccio] utilizza innanzitutto le forme brevi del racconto già in parte elaborate dall' autore del Novellino: sia quelle profane (dal fabliau al lai alla vida provenzale), sia quelle edificanti (dall'exemplum alla legenda alla visione); e inoltre si ispira a episodi 
isolati dei romanzi antichi e medievali. Ma oggetto di parodia possono essere, di volta in volta, anche la lirica stilnovistica, la predicazione e l'oratoria politica, la precettistica dei «libri di buoni costumi» e la letteratura odeporica; e poi, con particolare insistenza, la letteratura dei volgarizzamenti religiosi e tutte quante le molteplici espressioni della letteratura di pietà, fino ai settori che rasentano il folklore e la superstizione magica. $(174)^{15}$

Con la sua parodia multipla, in cui sono messi cioè in gioco molti generi contemporaneamente, la novella di Zima è certamente da considerarsi esemplare del gusto, o della profonda esigenza, del Boccaccio per l'ironizzazione e per i giochi di rimando, più o meno scoperti, ad altri autori. ${ }^{16}$ Bisogna ribadire, tuttavia, che i riferimenti ironizzanti o parodistici non compaiono sempre in virtù di una scelta cosciente. La riscrittura parodica boccacciana, cosciente o no, comporta comunque almeno tre modalità di intervento sul testo-modello: il capovolgimento, l'abbassamento e la concretizzazione (sia il capovolgimento che la concretizzazione possono ovviamente comportare l'abbassamento). Queste tre modalità, ricorrenti con eccezionale frequenza nella inventio del Decameron, sono attive nell'enigmatica e affascinante novella di Zima.

Se risulta ormai ben documentata la vasta gamma dei materiali usati da Boccaccio nella sua riscrittura parodica, molto lavoro rimane ancora da fare sulle tecniche impiegate, nonché sul significato delle parodie sia in singoli casi sia nel macrotesto boccacciano. L'attuale dibattito sulla serietà epistemologica del Decameron farebbe notevoli progressi se si riuscisse a rispondere alle cruciali questioni filosofiche sollevate dalle componenti parodiche dell'opera.

Nell'ambito di un esame complessivo della novella di Zima bisognerà infine tener conto anche di una componente autoparodica, o perlomeno autocitativa. Molti critici impegnati a indagare i fenomeni di ironizzazione nel Decameron hanno documentato gli elementi autoparodici dell'opera. Distinzione essenziale è tra l'autoparodia che guarda fuori dal libro (la riscrittura di segmenti di opere come il Teseida, il Filocolo, la Comedia delle ninfe fiorentine, l'Amorosa visione, brevemente ma efficacemente illustrata in Branca, "Introduzione" XXXV-XXXVIII) e l'autoparodia rivolta ad altri segmenti del libro stesso. A quest'ultimo tipo appartiene la pratica di Dioneo di presentare in gran parte delle giornate una novella parodica o di una storia specifica o del tema della giornata (Giannetto 14-18). Un caso di autoparodia che coinvolge racconti di diverse Giornate è la novella narrata da Filostrato nella Giornata $\mathrm{V}$ in risposta a quella narrata da Fiammetta nella IV. Nel caso della novella del Zima, l'autoparodia, o autocitazione, riguarda una delle opere giovanili dell'autore.

Nella parte $\mathrm{V}$ del Filostrato è collocato uno straordinario atto di riscrittura. Al fine di alleviare le proprie pene d'amore Troiolo compone una canzone in onore dell'amata Criseida. Il testo risulta essere un adattamento della canzone di Cino da Pistoia La bella vista e il bel guardo soave. Se può esserci 
disaccordo circa la natura parodica di questa riscrittura, non si può negare che si tratti di un'operazione di abbassamento. Pur tenendo conto che era nelle intenzioni di Boccaccio, quando scrisse il Filostrato, rendere più nobile il materiale narrativo dei cantari, siamo di fronte a un atto di abbassamento di rango letterario quando un componimento poetico come la canzone, il più nobile tra $\mathrm{i}$ metri della poesia in volgare, viene smembrato per forzarne le originarie stanze nelle griglie dell'ottava, il metro tradizionale della poesia popolare. È dunque possibile che la trasformazione di un sonetto di Cino in una novella possa essere collegata alla precedente trasformazione declassante della canzone di Cino in ottave popolari. Nella novella di Zima abbiamo osservato la trasformazione di un incontro mentale in un incontro reale, di un'astrazione lirica stilnovistica, il sonetto di Cino Ora che rise lo spirito mio, in una commedia erotica. La precedente riscrittura di Cino nel Filostrato suggerisce un ulteriore livello di lettura: la trasformazione di un sonetto di Cino in novella può essere anche la parodia della trasformazione di una canzone di Cino in ottave narrative. Tra le molte operazioni che Boccaccio compie con la novella di Zima, non è da escludere un atto di autoparodia riguardante la sua stessa pratica di riscrittura.

\section{The Johns Hopkins University}

\section{NOTE}

Questo saggio riproduce parzialmente e con qualche precisazione quanto compare in inglese nel quinto capitolo del mio volume Adventures in Speech: Rhetoric and Narration in Boccaccio's "Decameron". Philadelphia: U of Pennsylvania P, 1996. Per un prezioso lavoro di traduzione mi è grato ringraziare la dottoressa Federica Brunori del Department of Hispanic and Italian Studies a Johns Hopkins.

1 Così la Ferrante sull'importanza del tema del silenzio nella Giornata III: "Since deception and the exchange of rôles necessitate discretion on the part of the conscious participants, silence is a recurrent theme through the day: The gardener in 1 pretends to be a mute and the abbess maintains a discreet silence when she learns he is not; the groom in 2 must not speak when he visits the queen in the dark, and the king chooses not to speak about the affair; the lady in 3 tells no one but her confessor what is supposedly going on; the husband in 4 is enjoined to keep his special penance a secret; the wife in 5 is forbidden by her husband to speak; the wife in 6 cannot talk without revealing her identity, and the lover disguises his voice for the same reason; in 7, the dangers of speaking are disclosed - the wife says too much in her confession, and the murderers are discovered when they are overheard discussing the need to keep quiet about what they have done; in 8 , the wife is told to keep her husband's trip to Purgatory a secret; in 9 , as in 6 , the wife must be silent to conceal her identity" (593).

2 Zima riuscirà a capire i sentimenti della donna anche grazie agli eloquenti sguardi di lei. Si veda Ovidio, Ars Amatoria 1.574: "Saepe tacens vocem verbaque vultus habet".

3 Con questa strategia Zima introduce nella dichiarazione un elemento ludico che contribuisce a fiaccare le resistenze della donna. La dinamica psicologica di una tale situazione è tratteggiata da Freud con riferimento al motto di spirito: "Il pensiero cerca il travestimento dell'arguzia perché in tal modo si raccomanda alla nostra attenzione, può sembrarci più significativo, più valido, ma soprattutto perché questa veste corrompe e disorienta la critica. Noi siamo propensi ad attribuire al pensiero il merito di ciò che ci è piaciuto nella forma spiritosa; non 
propendiamo più a considerare errato qualcosa che ci abbia procurato diletto, col rischio di sciuparci una fonte di piacere. Se il motto $\mathrm{ci}$ ha fatti ridere, inoltre, si produce in noi la disposizione più sfavorevole alla critica [...]. Quando l'argomentazione cerca di tirare dalla sua parte la critica dell'ascoltatore, il motto mira a mettere in disparte questa critica" (Freud 119. 20). Si veda anche Olbrechts-Tyteca 11 . 11 potere persuasivo della giocosità e del comico era noto fin dall'antichità. Si veda la famosa sezione de risu nella Institutio Oratoria (6.3) di Quintiliano. Ovidio teorizza e consiglia l'uso della recitazione e delle arguzie come strumenti di seduzione (Ars Amatoria 3.367-68).

4 Sulla relazione tra narcisismo e bisogno di sedurre si veda Roccato 43-55.

5 Si veda Branca in Boccaccio, Decameron 372, nn. I, 3, 8; si veda inoltre Balduino 141-206.

6 Sulle forme poetiche dialogiche si veda Zumthor 357-74.

7 La modifica dell'espressione facciale secondo l'assunzione di ruoli differenti era ed è, ovviamente, cosa nota. Si veda Martelli 225-27.

8 La possibile connessione della novella di Zima con la Vita nuova di Dante è stata osservata per la prima volta, se non sbaglio, in Forni 72-74.

9 Per le connotazioni teatrali della novella si veda Baratto: “[...] il Zima sublima una passione e insieme strumentalizza una cultura: è costretto a un'ingegnosa commedia del linguaggio cortese e stilnovistico" (290). Muscetta parla di un "atto unico di commedia cortese" (214). Altre osservazioni sui momenti teatrali nel Decameron possono trovarsi in Baratto 239-69, Borsellino 11-50, Padoan 335-36, Russo 11-88.

10 Si confronti questo verso anche con il Fiore: "E di buona speranza il mi notrico" (3.13).

11 Di questi versi sono state proposte interpretazioni diverse (Orlando 103-4), in cui il possessivo di "sembiante" è attribuito al "pensero". Ritengo tuttavia che sia piú in linea con l'indole del Boccaccio l'interpretazione da me data.

12 Questo riferimento al Convivio non è sfuggito a Franco Fido (106, n.4), il quale l'ha semplicemente registrato come mai rilevato prima, senza attribuire ad esso piú ampia portata per il significato della novella.

13 "Branca uses the term 'ironization' rather than the term 'parody' because it seems better suited to render the idea of the type of parody that characterizes the Decameron, which, in general, is something somewhat hazy and which never extends to unrestrained comedy or caustic sarcasm. Viewed in this light, literary ironization, in particular, is seen by Branca not as a desecration of auctoritates, but as a simple 'forcing of linguistic and structural codes', that has as its aim 'the renewal from within of worn-out and consecrated themes and plots"' (Giannetto 19, n.2). Il termine parodia sarà da intendersi nella presente analisi in un senso che comprende quello attribuito dal Branca alla ironizzazione.

14 Non è questa la sede per trattare esaustivamente tutti i modi in cui Boccaccio ha utilizzato l'amatissimo capolavoro, con il quale non poteva fare a meno di misurarsi continuamente nello scrivere il suo. Basteranno qui tre esempi. In anni recenti, sia Robert Hollander che Luciano Rossi hanno riproposto l'ipotesi che dietro Ser Cepparello da Prato, il comico protagonista sulla soglia dell'opera (Decameron 1.1), ci sia il personaggio dantesco di Ser Brunetto Latini. Secondo Rossi il Boccaccio, colpito dalla lode che Dante elargisce al suo vecchio "maestro" sebbene egli figuri tra i dannati dell'Inferno, si prende la libertà di farne un "santo" nella sua prima novella, allo scopo di introdurre "il problema del rapporto verità-apparenza, che si rivelerà uno dei Leitmotive del Decameron" (388). Nell'Introduzione alla Giornata IV, in cui Bocaccio proclama che la fonte d'ispirazione della sua opera è da ricercarsi nelle donne in carne ed ossa, non nelle Muse, Rossi vede una "invocazione alla rovescia" in risposta alla invocazione di Dante alle Muse nel primo canto del Purgatorio (394-95). Non c'è bisogno di condividere fino in fondo la seguente radicale affermazione di questo studioso per riconoscerne l'indubbio valore critico: "Quel che nel Centonovelle è stato ribaltato è soprattutto la prospettiva per la quale i personaggi danteschi apparivano al poeta nella loro intima e immutabile essenza, così come avrebbero dovuto essere, talché era possibile "punirne i vizi» e «premiarne e meritarne le virtù». Gli 'eroi' boccacciani, al contrario, sono come appaiono, e le apparenze ne determinano di volta in volta l'essenza. Si pensi a Cepparello, 
Alatiel, Griselda" (382).

15 Si veda anche Branca 1976, "Introduzione" XXXIV-XXXV, a cui rinvia Delcorno.

16 Per la parodia multipla si veda Delcorno 174-75.

\section{OPERE CITATE}

Andreas Capellanus. On Love. Ed. Patrick Gerard Walsh. London: Duckworth, 1982.

Balduino, Armando. Boccaccio, Petrarca e altri poeti del Trecento. Firenze: Olschki, 1984.

Baratto, Mario. Realtà e stile nel Decameron. Vicenza: Pozza, 1970.

Borsellino, Nino. Rozzi e intronati. Esperienze e forme di teatro dal "Decameron" al "Candelaio». Roma: Bulzoni, 1974.

Boccaccio, Giovanni. Decameron. Torino: Einaudi, 1980.

-. Elegia di Madonna Fiammetta. Ed. Carlo Delcorno. In Tutte le opere. Ed. Vittore

Branca. Milano: Mondadori, 1994. 5.

Branca, Vittore. Boccaccio medievale e nuovi studi sul Decameron. Firenze: Sansoni 1986.

."Introduzione". Tutte le opere di Giovanni Boccaccio. Vol. 4. Decameron. Ed. Vit-

tore Branca. Milano: Mondadori, 1976. XI-XXXV111.

Cino da Pistoia. Poeti del Dolce Stil Nuovo. Ed. Mario Marti. Firenze: Le Monnier, 1969. 421923.

Dante Alighieri. Il Convivio. Ed. Giovanni Busnelli e Giuseppe Vandelli. 2 voll. Firenze: Le Monnier, 1968.

-Il fiore. Ed. Gianfranco Contini. Dante Alighieri. Opere minori. Ed. Domenico De Robertis e Gianfranco Contini. Milano-Napoli: Ricciardi, 1984. 1.1.553-798.

Vita nuova. Ed. Domenico De Robertis. Milano: Ricciardi, 1980.

Delcorno, Carlo. "Ironia/Parodia". Lessico critico decameroniano. Ed. Renzo Bragantini e Pier Massimo Forni. Torino: Boringhieri, 1995. 162-91.

De Robertis Domenico. "Storia della poesia e poesia della propria storia nel XXII della "Vita nuova»". Studi danteschi 51 (1978): 153-77.

Faral, Edmond. Les jongleurs en France au moyen âge. Paris: Champion, 1910.

Ferrante, Joan M. "Narrative Patterns in the Decameron." Romance Philology 31 (1978): 584604.

Fido, Franco. Il regime delle simmetrie imperfette. Studi sul "Decameron». Milano: Angeli, 1988.

Forni, Pier Massimo. "Zima sermocinante («Decameron», 111 5)”. Giornale Storico della Letteratura Italiana 163 (1986): 63-74.

Freud, Sigmund. "Il motto di spirito e la sua relazione con l'inconscio". Opere. 1905-1908. Torino: Boringhieri, 1972. 1-211.

Giannetto, Nella. "Parody in the Decameron: a Contented Captive and Dioneo". The Italianist 1 (1981): 7-23.

Martelli, Mario. "Considerazioni sulla tradizione della novella spicciolata". La novella. 1. 215-44.

Muscetta, Carlo. Boccacccio. Bari: Laterza, 1981.

La novella italiana. Atti del convegno di Caprarola. 19-24 settembre 1988. 2 voll. Roma: Salerno, 1989.

Olbrechts-Tyteca, Lucie. Il comico del discorso. Un contributo alla teoria generale del comico e del riso. Milano: Feltrinelli, 1977.

Orlando, Sandro. Appunti sul Dolce stil nuovo: Testi commentati ad uso dell'esame di Filologia romanza, a.a. 1986-1987. Alessandria: Edizioni dell'Orso, 1987.

Ovid. The Art of Love and Other Poems. Trans. John Henry Mozley. Cambridge, Mass.: Harvard UP, 1985.

Padoan, Giorgio. "Il senso del teatro nei secoli senza teatro". Concetto, storia, mito e immagini del Medio Evo. Ed. Vittore Branca. Firenze: Sansoni, 1973. 325-38.

Roccato, Paolo. "La seduzione come relazione collusiva." La seduzione: saggi psico-analitici. Ed. Anteo Saraval. Milano: Cortina, 1989. 43-55. 
Rossi, Luciano. "Ironia e parodia nel Decameron: da Ciappelletto a Griselda." La novella. 1.365405.

Russo, Vittorio. Con le muse in Parnaso. Napoli: Bibliopolis, 1983.

Suitner, Franco. La poesia satirica e giocosa nell'età dei comuni. Padova: Antenore, 1983.

Zumthor, Paul. Toward a Medieval Poetics. Minneapolis-London: U of Minnesota P, 1992. 